\title{
La ciudad real, la ciudad imaginada, la ciudad deseada. Las representaciones de la ciudad y los mapas mentales como expresiones de la complejidad
}

PABLO SANDOVAL CABRERA ${ }^{1}$

\section{Resumen}

El trabajo que aquí se presenta constituye un breve e inicial ensayo que, en principio, representa un esfuerzo por incursionar en el debate teórico sobre la génesis de las percepciones sociales, argumentando, a la luz de la teoría existente, que dichas percepciones son producto de un proceso cognitivo complejo en que se entrelazan, a diversas escalas, la conciencia y lo social-cultural en una relación entre lo simbólico y lo imaginario, en un marco interfacético de cognición del sujeto que intenta aprehender la imagen del objeto (fenómeno) observado, en su complejidad, poniendo en juego su experiencia, cultura, valores sociales y capacidades diversas de percepción, comprensión, relación e integración.

Clasificación JEL: O21, P25, R11, R23

Fecha de recepción: 25 de mayo de 2020. Fecha de aceptación: 9 de junio de 2020.

1. Profesor investigador, Departamento de Economía, Centro Universitario de Ciencias Económico Administrativas de la Universidad de Guadalajara. Dirección electrónica: casapa2636@gmail.com. 


\section{THE REAL CITY, THE IMAGINED CITY, THE DESIRED CITY. REPRESENTATIONS OF THE CITY AND MIND MAPS AS EXPRESSIONS OF COMPLEXITY}

\section{Abstract}

The work presented here constitutes a brief and initial essay which, in principle, represents an effort to enter the theoretical debate on the genesis of social perceptions, arguing in the light of existing theory that they are the product of a complex cognitive process in which consciousness and the social-cultural are intertwined, at various scales, in a relationship between the symbolic and the imaginary, in an interfacial framework of cognition of the subject trying to apprehend the image of the observed object (phenomenon), in its complexity, putting into play their experience, culture, social values and diverse capacities of perception, understanding, relationship and integration.

In particular, it is interesting to highlight that its elaboration starts from the instituted, the underlying, the presupposed and the socially and symbolically constructed. From this origin, the different forms of individual and collective representation of the different phenomena that derive in the socio-cultural dynamics of cities are explained.

After a brief theoretical analysis of what is defined as complexity in its relation to social perceptions, a task that is not simple and barely outlined in this essay, we move on to the description of one of the frequently used techniques for the subjective construction of the city, I mean the technique (or method) of mind maps.

For this purpose, the work is divided into four sections. The first of them deals with the analysis of the theory of complexity, the imaginaries and their relationship with the construction of social representations. In a second section, the analysis of social representations is approached from the perspective of urban sociology and the existing literature on the subject of mind maps, starting with, perhaps, the first study published on it, from the pen by K. Lynch in the year 1960 and which is entitled "The image of the city." Other authors are reviewed and the analysis of narrative literature is suggested as a culturally very attractive source on the subject under study. In this regard, and just as an example, the novel by Ítalo Calvino entitled "The Invisible Cities" is reviewed, which stands out as a highly creative literary exercise of subjective reconstruction of cities.

In the third part of the work, the technical and methodological aspects related to the use of mental maps in urban research are reviewed. Among other things, the application of the technique, the selection criteria of the study universe and the method are highlighted, as well as the advantages and disadvantages of its application. The purpose of this section is to illustrate the process that occurs when individuals graphically represent the city. It undoubtedly combines cognitive and perception aspects, as well as sociocultural elements that end up producing a very diverse image of the city, very close to the processes of social anchoring and objectification / objectification, which Moscovici (1986) recognizes in the complex construction of social representations. 
The last part of the study addresses the analysis of an application experience of mental maps highlighting its methodological aspects, its scope and results. The work analyzed in this section was carried out by Martha de Alba and is entitled "Mental maps of Mexico City, a psychosocial approach to the study of spatial representations", which was published by the Universidad Autónoma Metropolitana, Iztapalapa unit.

It is recognized that in so few pages the approach to a theoretical, methodological and even philosophical topic of singular importance is barely outlined, such as the study of complexity in its relation to social representations, as well as the use of the technique of mental maps. Despite this, the purpose of proposing an analysis structure and a follow-up line of the topic for future research is fulfilled.

\section{Introducción}

El trabajo que aquí se presenta constituye un breve e inicial ensayo que, en principio, representa un esfuerzo por incursionar en el debate teórico sobre la génesis de las percepciones sociales, argumentando, a la luz de la teoría existente, que dichas percepciones son producto de un proceso cognitivo complejo en que se entrelazan, a diversas escalas, la conciencia y lo social-cultural en una relación entre lo simbólico y lo imaginario, en un marco interfacético de cognición del sujeto que intenta aprehender la imagen del objeto (fenómeno) observado, en su complejidad, poniendo en juego su experiencia, cultura, valores sociales y capacidades diversas de percepción, comprensión, relación e integración.

En lo particular, interesa destacar que su elaboración parte de lo instituido, lo subyacente, lo pre-supuesto y lo social y simbólicamente construido. A partir de este origen se explican las diferentes formas de representación individual y colectiva de los distintos fenómenos que derivan en la dinámica socio cultural de las ciudades.

Después de un breve análisis teórico sobre lo que se define como complejidad en su relación con las percepciones sociales, tarea nada sencilla y apenas esbozada en este ensayo, se transita a la descripción de una de las técnicas de frecuente utilización para la construcción subjetiva de la ciudad; me refiero a la técnica (o método) de los mapas mentales.

Con este propósito, el trabajo se divide en cuatro apartados. En el primero de ellos se aborda el análisis de la teoría sobre complejidad, los imaginarios y su relación con la construcción de las representaciones sociales. En un segundo apartado se aborda el análisis de las representaciones sociales desde el enfoque de la sociología urbana y la literatura existente sobre el tema de los mapas mentales, y se inicia con, tal vez, el primer estudio que se publicó sobre dicho tema, de la pluma de K. Lynch en el año de 1960 y que llevo por título "La imagen de la ciudad". Se revisan otros autores y se sugiere el análisis de la literatura narrativa como una fuente culturalmente muy atractiva sobre el tema en estudio. Al respecto, y solo como ejemplo, se revisa la novela de Ítalo Calvino titulada Las ciudades invisibles, la cual se destaca como un ejercicio literario sumamente creativo de reconstrucción subjetiva de las ciudades. 
En la tercera parte del trabajo se revisan los aspectos técnicos y metodológicos relacionados con el uso de los mapas mentales en la investigación de lo urbano. Se destacan, entre otros aspectos, la aplicación de la técnica, los criterios de selección del universo de estudio y el método, así como las ventajas y desventajas de su aplicación. El propósito de este apartado es ilustrar el proceso que ocurre cuando los individuos representamos gráficamente la ciudad. En él se conjugan, sin duda, aspectos cognitivos, de percepción, así como de elementos socioculturales que terminan por producir una muy diversa imagen de la ciudad, muy cercana a los procesos de anclaje social y objetificación/objetivación, que Moscovici (1986) reconoce en la compleja construcción de las representaciones sociales.

En la última parte del estudio se aborda el análisis de una experiencia de aplicación de los mapas mentales y se destacan sus aspectos metodológicos, sus alcances y resultados. El trabajo analizado en este apartado fue realizado por Martha de Alba, y lleva por título "Mapas mentales de la Ciudad de México una aproximación psicosocial al estudio de las representaciones espaciales", el cual fue publicado por la Universidad Autónoma Metropolitana, unidad Iztapalapa.

Se reconoce que en tan pocas páginas apenas se bosqueja el abordaje de un tema teórico, metodológico e incluso filosófico de singular importancia, como lo es el estudio de la complejidad en su relación con las representaciones sociales, así como el uso de la técnica de los mapas mentales. A pesar de ello, se cumple con el propósito de proponer una estructura de análisis y una línea de seguimiento del tema para futuras investigaciones.

\section{La compleja definición de la complejidad}

\section{A) Que se entiende por complejidad}

El pensamiento occidental moderno, inspirado en la filosofía clásica (con Platón y Aristóteles como sus principales representantes) aún dominante, ha asumido distintas formas y retóricas en línea con la corriente racionalista y sus derivados, tales como el discurso modernizador en sociología y la racionalidad instrumental en economía, pero todas ellas circunscritas en lo que E. Morín denomina el paradigma de simplificación.

Dicho paradigma intenta fragmentar, idealizar, racionalizar y normalizar todos los fenómenos, limitando su contexto, significado y naturaleza a esquemas, procesos, algoritmos y sistemas invariantes. Esta forma de ver el mundo encuentra muy diversas expresiones en la praxis social y política, así como en la ética que la sustenta, lo que determina la visión que tenemos sobre el mundo y el lugar que en él ocupa el ser humano.

Dicha perspectiva epistemológica apuesta por una supuesta objetividad en la que se excluye al sujeto, lo que desemboca en una inaceptable simplificación del proceso cognitivo y del conocimiento generado.

Como señala Nudler (2009) desde su teoría de los espacios controversiales, a partir de la puesta en juego de la diversidad de pensamientos y experiencias que pueden 
ser puestos en discusión entre sujetos es posible superar el aporte restrictivo del método científico tradicional en su linealidad extrema, donde pareciera que el método garantiza la verdad cuando ella está es en la complejidad de las relaciones y los comportamientos de los agentes considerados, en sus "mundos de la vida".

En correspondencia con Nudler, la visión tradicional dominante es confrontada y puesta en entredicho por el paradigma de la complejidad a partir de principios radicalmente distintos, no solo en el campo del conocimiento sino también en la praxis social y política (Soto, 1999). Para lograr el cometido es necesario señalar algunas de las particularidades que, en todo caso, podrían servir de elementos identitarios de la complejidad (pensamiento, sistema, o fenómeno):

1. "La complejidad es una palabra problema y no una palabra solución" (ibid: 3), lo que significa que es un desafío transitar el camino de la complejidad. En este sentido, el pensamiento complejo se convierte, en sí mismo, en un conjunto de alternativas para intentar afrontar dicho desafío, en lugar de evadirlo.

2. Complejidad es incompletitud, incertidumbre y apertura. Es un pasaje abierto, inconmensurable e incierto.

3. Es relación y apertura en tanto acepta diferentes lecturas de la realidad, sin negar su necesario encuentro.

4. La complejidad pretende conocer el sujeto y empoderarlo a través del propio conocimiento de los elementos que lo condicionan.

5. Lo complejo se explica por comportamientos no lineales y diversos grados de libertad.

6. Su dinámica, en el marco de un sistema, se expresa en múltiples relaciones de interdependencia entre sus componentes.

7. Es imposible comprender su dinámica y estructura a partir del análisis particular de sus componentes (el todo es más que la suma de sus partes). Equivale a "lo que Luhmann denomina ausencia de realidad óntica en los hechos aislados" (De la Reza: 90) en el sentido en que ellos solo adquieren significado en el marco del sistema al que se adscriben.

8. En el marco de un sistema, la complejidad no se manifiesta absolutamente apegada al orden, pero tampoco es absolutamente caótica.

Por estas características, el estudio de los sistemas complejos debe ser abordado desde una óptica múltiple, integral y holística; es decir, transdisciplinar, considerando que "los comportamientos no lineales son recurrentes y aparecen jerarquías de niveles de descripción, así como propiedades que emergen de comportamientos colectivos" (Cocho y Martínez, 2013: 49)

En general, los sistemas que son complejos no son difíciles de reconocer e identificar, se encuentran entre otros: "la turbulencia, los huracanes, los sismos, una célula, el sistema nervioso o el inmunológico, el desarrollo urbano, el sistema de salud o el de seguridad pública, la evolución de ecosistemas, la dinámica de los mercados, de la bolsa de valores, la mente, la conciencia y el lenguaje" (ibid: 48). 
Los procesos de elaboración de las representaciones sociales son ejemplos de fenómenos complejos al momento en que conjugan niveles de intervención relacionados con lo cognitivo y la conciencia en un entramado de interacciones con lo subyacente imaginado y el entorno social, cultural y simbólico en que se desenvuelve el individuo. Al respecto, Díaz (2013: 194) afirma que:

la conciencia, como proceso complejo, se manifiesta al momento en que la información ascendente (bottom-up) de los niveles neurobiológicos inferiores converge con la información descendente (top-down) de niveles sociales y culturales pues, para que ocurra un evento consciente, se requiere tanto de una activación de los sustratos cerebrales a partir de su nivel molecular y que afecta de manera progresiva a los niveles de actividad neuronal, redes de neuronas, núcleos y sistemas, como el concurso de la información descendente de los sustratos sociales o culturales para determinar su contenido.

\section{Destaca que:}

la conciencia, al ser el aspecto fenomenológico del emergente más encumbrado de los sistemas cerebrales, este adquiriría no solo vigorosas capacidades de navegación en el encéfalo (...) además influirá en una cadena de eventos nerviosos que desemboquen en cambios fisiológicos, comportamientos, expresiones y acciones (Díaz: 193),

las cuales afectarán el entorno social y cultural al actuar sobre él, y generan nuevas elaboraciones de la realidad mediante el arte, la cultura, la técnica y la ciencia, e incluso dará forma a nuevas formulaciones o representaciones de dicho entorno.

Roger Bartra (2005) se refiere a este proceso de interacción simbólica entre el medio social y la conciencia, como el "exocerebro", entendiendo que "la conciencia no es concebible sin el concurso histórico, fáctico, sistemático, consonante y constante de ambas esferas, la cerebral de índole biológica y la cultural de carácter social" (Díaz, 2013: 194).

A partir de la conjugación e interacción a distintos niveles, entre lo biológico, lo psicológico y lo social, "la conciencia adquiere características tan sorprendentes como la libertad de acción, la capacidad de conocimiento o la introspección” (ibidem).

\section{B) Las representaciones sociales (RS) como procesos complejos (PC)}

Es necesario reconocer en principio que "representar es un acto de pensamiento mediante el cual un sujeto le da significado a un objeto que puede ser real o imaginario" (Caravaca, 2017: 3). Por lo tanto, la representación no puede ocurrir sin la existencia de ambos, y esta adquiere sentido al "contener" a ellos en sí misma. Este proceso de representación sintetiza antagónicamente diversos saberes, ideas, valores y percepciones sobre la realidad cotidiana, los que al confluir dan forma a lo que Moscovici (1986) llama polifasia cognitiva, fenómeno en el que se originan las representaciones sociales, que hacen de ellas un mosaico diverso, contrastante y dinámico, resultado de un proceso naturalmente complejo. 
De acuerdo con Moscovici (citado por Caravaca, 2017), los procesos generadores de las representaciones sociales son de dos tipos, ambos interrelacionados simbióticamente.

El primero se denomina "anclaje social" y consiste literalmente en "anclar" o fijar un fenómeno novedoso en categorías referenciales preexistentes. Al ser un fenómeno inusitado, este tiende a clasificarse, ordenarse y categorizarse a partir de significados ya existentes e interiorizados. Esto es, las nuevas representaciones sociales se enmarcan en lo ya "archivado", y con ello el proceso de cognición se da a partir de lo instituido.

Lo instituido se fundamenta en los valores, símbolos y significados ya validados social y culturalmente. En este sentido, el anclaje social hace posible la materialización físico-simbólica de lo nuevo; es decir de la representación social emergente. Este proceso de materialización se conoce como objetificación/objetivación de la nueva representación social.

El proceso de objetificación/objetivación ocurre en paralelo al "anclaje social"; consiste en organizar los elementos de una representación social, así como en otorgarle una materialidad físico-simbólica.

La manifestación del saber o cognición social como proceso complejo finalmente se expresa como un todo dinámico en el que confluyen aspectos intrapersonales, interpersonales e ideológicos, los cuales adquieren forma en las representaciones sociales gracias a los procesos de anclaje social y objetificación/objetivación del fenómeno nuevo.

Las representaciones sociales explican los orígenes del saber social en la medida en que auxilian en la comprensión del origen de los esquemas cognitivos, al tiempo que iluminan la génesis de sus atribuciones y anclajes socioculturales (ibid). Constituyen, en sí mismas, conocimiento nuevo (signos, símbolos, conceptos, imágenes, etcétera), pero en su proceso de elaboración cognitiva superan a dichos elementos, al ser el resultado de procesos complejos por naturaleza.

\section{El imaginario y las representaciones sociales como sustento para la construcción subjetiva de la ciudad}

Ciudad, imaginario, representaciones sociales y mapa mental constituyen términos inmanentemente articulados; encierran, sin embargo, una singular complejidad en su abordaje.

La ciudad como objeto de estudio debe ser, en principio, concebida como un ente vivo en el que sus habitantes le dan sentido, a partir de significados construidos por las interacciones manifiestas bajo diferentes formas de expresión y comunicación que la propia sociedad crea y recrea a través del tiempo.

La ciudad, dice Amendola (2000: 101):

no se constituye solo por el espacio de la función, de la previsión y de la causalidad, sino también por aquel de la casualidad, del azar y de la indeterminación. En el paseo se revela 
la posibilidad de explorar la ciudad en numerosas direcciones, encontrando cada vez nuevos significados, épocas, símbolos, proyectos colectivos y personales.

Las representaciones sociales son algo más que simples productos de la mente, son fundamentalmente elaboraciones simbólicas que se construyen y deconstruyen en un ambiente de interacciones sociales; por tanto, son una obra colectiva. En este sentido, "las representaciones sociales constituyen producciones simbólicas que se construyen a partir de imaginarios sociales en los cuales se representa y valora colectivamente un hecho o un fenómeno de la vida social" (Morales, 2014).

La geografía de la percepción ha sido esencial para el análisis urbanístico y se enraíza teóricamente en los trabajos realizados por la escuela de Chicago con obras como la de Kevin Lynch (1960), The Image of the City, aunque con fuertes cimientos en los trabajos de Park (1999), quien concebía la ciudad como un ente ordenado, pero altamente dinámico a través de instituciones que determinan las condiciones de la vida urbana y le dan una connotación ecológica a la vida en las ciudades. El mismo autor enfatiza que

Una de las cosas que hacen a la ciudad un lugar particularmente favorable para el estudio de las instituciones y de la vida social en general, reside es el hecho de que, bajo las condiciones de la vida urbana las instituciones se desarrollan rápidamente. Crecen ante nuestros ojos: los procesos de su desarrollo son accesibles a la observación y, eventualmente a la experimentación (Park: 126).

Park es, sin duda, uno de los mayores impulsores del estudio de la ciudad a partir de su inmersión en ella, mediante las técnicas de la observación e incluso la experimentación. Solo así es posible entender la naturaleza viva y subjetivamente construida de la ciudad y sus complicados procesos.

Igual relevancia tienen los trabajos de Simmel (1986) y Lefebvre (1969), quienes conciben las sociedades como "entes" elaborados y reelaborados a partir del movimiento constante de asociaciones, negociaciones y contradicciones a veces imperceptibles pero definitivas en la determinación de la dinámica de las ciudades, incluso más allá de sus fronteras, matizaría el propio Simmel. Dichas interacciones sociales encuentran su asidero en la inevitable confrontación entre lo subjetivo, relacionado con las emociones y el espíritu, y lo objetivo, manifestado a través de las conductas externas, y por tanto observables, de los individuos en su interacción social.

Sin embargo, el concepto más nítido de representación social, tal vez, debe su formulación a Moscovici (1961), reconociendo también un antecedente importante en lo que Durkhein (1898) denomina "representación colectiva". Este concepto resalta aquellas formas de conocimiento socialmente construidas.

El término representaciones sociales, además de elaboraciones mentales, referentes simbólicos que se convierten en mecanismo de interpretación y significación de la realidad, base fundamental en la determinación de las relaciones entre los sujetos, en algo que podría aproximarse a lo que normalmente se entiende por sentido común (Moscovici, 1981: 181). 
Lo más relevante del concepto representaciones sociales es que, por su contenido simbólico, no solamente permite conocer o aprehender la realidad social, sino también, adicionalmente, le otorga sentido a dicha realidad.

Para Jodelet (1986), inscrita en la misma corriente de la psicología social impulsada por Moscovici, "las representaciones sociales" pueden adoptar diversas formas:

imágenes que condensan un conjunto de significados, sistemas de referencias, para interpretar lo que nos sucede, categorías para clasificar las circunstancias, fenómenos e individuos con quienes debemos tratar y teorías para establecer hechos sobre ellos. La noción de representación social nos sitúa en el punto donde se articula lo psicológico con lo social.

Y agrega: "La representación social no es un duplicado de lo real o de lo ideal, ni la parte subjetiva del objeto, ni la parte objetiva del sujeto: es la relación del hombre con las cosas y con los demás hombres".

Sus componentes o características esenciales le dan una connotación mucho más clara y precisa, estas son las siguientes, de acuerdo con Jodelet (ibid).

a) Siempre es la representación de un objeto, persona, acontecimiento, ideal, etc. Por eso se llama representación.

b) Tienen un carácter de imagen y la propiedad de poder intercambiar lo sensible y la idea, la percepción y el concepto. La forma de representarnos el objeto, la persona, etc., es mediante una imagen de ella.

c) Tienen un carácter simbólico y significante. La representación como imagen, concepto, etc., no es una mera representación de objeto ausente, sino una construcción, donde el sujeto aporta algo creativo. Es decir: a) Tiene un carácter constructivo, b) Tiene un carácter autónomo y creativo.

Son estas características, delimitadas por Jodelet, las que permiten entender que, aun siendo construidas, las representaciones sociales, necesaria e innegablemente de naturaleza colectiva, son construcciones mentales que los individuos realizan de su entorno urbano.

La ciudad como una construcción simbólica se relaciona "funcionalmente" con otro concepto importante que nutre, hace posible y cimienta esta posibilidad. Sollors (1989, citado por Safa, 2000) sostiene que:

en el terreno de los imaginarios, las ciudades imaginadas, soñadas, percibidas como posibles, se convierten en un objeto de estudio que, en las actuales condiciones de los contextos de la megalópolis, pueden ser muy pertinentes (...) Y con ello, comprendemos nuevamente que siempre habrá ciudades metafóricas, ciudades superpuestas a las reales, pero no por ello menos importantes.

Esto significa que lo imaginario constituye lo presupuesto, lo subyacente que les da sentido a las construcciones mentales que hacemos de la ciudad.

Castoriadis (1975: 220) plantea que el imaginario supera en elaboración y en consecuencia a aquello de lo cual se nutre, y ello es lo significativo y complejo del imagi- 
nario: "si dijimos que el simbolismo presupone lo imaginario radical, y se apoya en él, no significa que el simbolismo no sea, globalmente, sino imaginario en su contenido". Una sociedad existe "en tanto plantea la exigencia de la significación como universal y total, y en tanto postula su mundo de las significaciones como aquello que permite satisfacer esta exigencia (ibid: 312 ).

Desde esta perspectiva, una colectividad de seres humanos interrelacionados no se identifica solo por sus condiciones materiales de vida y reproducción, sino también, y fundamentalmente, por el conjunto de significaciones, percepciones y representaciones de la realidad que sus miembros son capaces de idealizar y construir individual y colectivamente.

Su asidero está en los imaginarios que la propia sociedad ha instituido; la amplitud y excelsitud de las significaciones representadas se sostienen, necesariamente, en el imaginario. Es decir, las posibilidades de representatividad de la sociedad como un proceso de creación y recreación de sí misma están fundadas, cimentadas y delimitadas en el imaginario social. $\mathrm{Al}$ respecto, Cabrera señala que:

el imaginario no es imagen, sin condiciones de posibilidad y existencia para que una imagen sea imagen de y porque no "denota" nada y lo "connota" todo no puede ser captado de manera directa sino derivada, como el centro invisible de lo real-racional-simbólico que constituye toda la sociedad y que se hace presente en la conducta de los pueblos y de los individuos.

De forma por lo demás elocuente, Lizcano (2003) resalta la importancia de los imaginarios en la elaboración individual y colectiva de representaciones de la vida social, al concebir que "el imaginario educa la mirada, una mirada que no mira nunca directamente las cosas; las mira a través de las configuraciones imaginarias en las que el ojo se alimenta" (Lizcano, 2003: 4). En este sentido, el imaginario no se manifiesta nítidamente en sí, sino para sí. Esto es, son el presupuesto de la mayoría de nuestras ideas, percepciones y actos, porque condicionan nuestro ser y actuar, pero no aparecen a la "luz" como manifestación especialmente expresada, "el imaginario en que cada uno habitamos, el imaginario que nos habita, nos obstruye así ciertas percepciones, nos hurta ciertos caminos, pero también pone gratuitamente a nuestra disposición toda su potencia, todos los modos del poder ser de los que él está preñado" (ibid: 6).

La concepción de la sociedad y de la ciudad que habita como "creación y creación de sí misma, autocreación, es la emergencia de una nueva forma ontológica - un nuevo ciclo- y de un nuevo nivel y modo de ser. Es una cuasi totalidad cohesionada y por las significaciones que otras instituciones encarnan" (Castoriadis, 1997: 4). Es, entonces, una creación esencialmente social. Y dicha creación tiene su origen en el imaginario, el cual nutre las propias posibilidades de construcción individual y social. 


\section{Los mapas mentales como un recurso de representación de lo percibido, (geografía mental)}

\section{A) Qué son los mapas mentales}

Los mapas mentales constituyen un instrumento novedoso de investigación para indagar cómo los individuos perciben el entorno que los rodea, reconociendo, como explicamos en el apartado anterior, que estas percepciones tienen su fundamento en el imaginario.

El antecedente más notable de construcción subjetiva de la ciudad se remonta al análisis urbanístico de la Escuela de Chicago, bajo el liderazgo de Kevin Lynch, cuya obra más reconocida, publicada en 1960, lleva por título La imagen de la ciudad.

En su obra, Lynch trata de captar subjetivamente la ciudad vivida dando lugar a una perspectiva de análisis de lo urbano sustentado en lo fenomenológico, lo vivencial y lo humano.

Se reconoce que cada individuo tiene una imagen mental de la ciudad influida por el lugar donde habita, su área circundante y los entornos que recorre para realizar las distintas actividades que le exige la vida urbana, e incluso por la cultura y la influencia que ejercen los medios de comunicación.

En este sentido, es importante reconocer que el espacio subjetivo que se expresa mediante los mapas mentales es diferente al espacio territorial que recorren y conocen los individuos. Por esta razón, es muy común que para un mismo entorno espacial haya manifestaciones, representadas por los mapas mentales, significativamente diferentes.

Los mapas mentales asumen distintas formas de representación. Pueden ser una representación cartográfica, figuras, nombres, entre otros, o una combinación de ellos.

Las posibilidades de elaboración de los mapas mentales derivan del tiempo disponible, el acceso a información, la propia movilidad (incluyendo el medio utilizado) e incluso de la aplicación de estímulos.

De acuerdo co K. Lynch (op. cit: 61-98), son los elementos del paisaje los que otorgan cierta regularidad en la elaboración de los mapas de la ciudad. Estos son:

1. Hitos: entendidos como referencias específicas o puntos destacados en el plano: monumentos, edificios, museos, etcétera.

2. Sendas: son conductos que sigue el observador normal, ocasional o potencialmente: calles, senderos, canales, vías férreas, entre otros.

3. Bordes: constituyen los límites entre dos fases o rupturas lineales de la continuidad, como los límites físicos que separan un barrio o colonia de otro.

4. Barrios: son las subdivisiones de una ciudad social e identitariamente reconocidos por los individuos.

5. Nodos: son puntos de confluencia regularmente estratégicos de una ciudad en los que puede darse una confluencia o cruce de sendas, conclusión de una ruta o una ruptura en el transporte. Adquieren distintas dimensiones e incluso la ciudad 
puede ser considerada como nodo si la escala del observador es nacional o internacional.

6. Mojones: constituyen puntos de referencia externos al observador. Se destacan por la singularidad que el propio observador les otorga por sus características físicas, y pueden cumplir la función de referentes guía en sustitución de ciertas continuidades. Tal es el caso de ciertas figuras arquitectónicas que sobresalen en el contexto edificado de la ciudad.

En los estudios más recientes que se han elaborado se comprueba que existen diversas condiciones socioambientales que inciden en las percepciones que los observadores hacen de la ciudad. Entre otras, el género, la edad, el origen étnico, las condiciones de hábitat, condiciones económicas, entre otros.

Una experiencia destacable en la indagatoria realizada para abordar el estudio de los mapas mentales fue comprobar que la literatura es un recurso excepcional para percibir y narrar la ciudad.

No es la intención de este artículo realizar una revisión exhaustiva sobre la función que cumple la literatura en este ámbito, solamente haré referencia a la excelente novela escrita por Ítalo Calvino que lleva por título Las ciudades invisibles.

En dicha obra, el autor, nacido en Santiago de Cuba en 1923, de origen italiano, tal vez sin pretenderlo, elabora en forma magistral, con las bondades y restricciones que otorga la narrativa, una rica sucesión de "mapas mentales" de las ciudades vividas, las ciudades deseadas, las ciudades de ensueño que son interactivamente narradas por el explorador Marco Polo al emperador de los tártaros, el gran Kublai Kan.

Haciendo un uso genial de la metáfora, Calvino da vida en su narración a una amplia multitud de ciudades, todas con nombre de mujer, que fueron conocidas, pensadas o soñadas, en los múltiples viajes que Marco Polo realizó alrededor del mundo.

Las ciudades invisibles constituyen una sucesión de ciudades narradas denominadas intencional y referencialmente de muy diversas formas: Las ciudades y la memoria, las ciudades y el deseo, las ciudades y los signos, las ciudades y los trueques, las ciudades y los ojos, las ciudades y los muertos, etcétera.

En esencia, la novela de Calvino deriva de uno o varios ejercicios creativos, en una especie de viajes por la imaginación, en las que el autor narra las características físicas, los cánones de convivencia, los deseos y los significados que influyen en la dinámica de cada ciudad.

Sin duda su obra es lo más próximo a la elaboración de diversos mapas mentales descritos de manera magistral por su pluma.

En algunos momentos Calvino se parece más a un sociólogo o urbanista que a un literato. Su novela está llena de referencias invaluables para entender lo que diversos estudiosos de la ciudad han planteado con relación a los significados que adquiere la ciudad en la medida en que es social y subjetivamente construida.

En un excelente pasaje de la novela el autor descubre sus propósitos literarios. 
Lo que importa a mi Marco Polo es descubrir las razones secretas que han llevado a los hombres a vivir en las ciudades, razones que puedan valer más allá de todas las crisis. Las ciudades son un conjunto de muchas cosas: memorias, deseos, signos de un lenguaje; son lugares de trueque, como explican todos los libros de historia de la economía, pero estos trueques no lo son solo de mercancías, son también trueques de palabras, de deseos, de recuerdos (Calvino: 16).

Cada elemento constitutivo, estructural o vivo, de la ciudad es ingeniosamente descrito por el autor, denominado metafóricamente por su rasgo más distintivo.

En la ciudad de los signos (Tamara) "el ojo no ve cosas sino figuras de cosas, que significan otras cosas", en una especie de encadenamientos simbólicos nacidos del arraigado imaginario o de lo instituido por el imaginario, para decirlo en el lenguaje de Castoriadis.

Esta obra de Calvino constituye una sucesión de interpretaciones y representaciones de la ciudad, o las ciudades para ser más preciso, más cercano a un análisis sociológico que a una novela.

En lo que sigue haremos referencia a un conjunto de citas, no necesariamente articuladas, con el único propósito de evidenciar más al sociólogo que al literato:

La memoria es redundante: repite los signos para que la ciudad empiece a existir (p. 28).

La metrópoli tiene este atractivo más: que a través de lo que ha llegado a ser se puede evocar con nostalgia lo que fue (p. 33).

de cada ciudad que Marco le describía, la mente del gran Kan partía por cuenta propia y desmontaba la ciudad parte por parte, la reconstruía de otro modo, sustituyendo ingredientes, desplazándolos, invirtiéndolos (p. 41).

las ciudades como los sueños, están construidas de deseos y de temores, aunque el hilo de su discurrir sea secreto, sus normas absurdas, sus perspectivas engañosas, y cada una esconda otra (p. 42).

No se debe confundir nunca a la ciudad con las palabras que la describen. Y sin embargo entre la una y la otra hay una relación (p. 52).

\section{B) Aspectos técnico metodológicos relacionados con los mapas mentales}

La metodología de los mapas mentales se presenta como una alternativa práctica de aproximación para la comprensión de los procesos de construcción de las representaciones sociales, propósito con el que aquí se revisan. Para cumplir con este cometido es necesario especificar sus elementos metodológicos esenciales:

1. Universo de estudio. El universo de estudio en las investigaciones sobre percepciones psicosociales de la ciudad mediante la aplicación de mapas mentales lo constituye los habitantes de la ciudad en estudio. Regularmente se eligen muestras de habitantes sin necesidad de representación estadística, algunas veces estableciendo algún criterio sociocultural (edad, sexo, condición económica etcétera), dependiendo de los propósitos de la investigación.

2. En qué consiste la técnica. Se les pide a los participantes que elaboren representaciones cartográficas de la ciudad (u otra dimensión del territorio urbano), algo 
parecido a un dibujo en el que destaquen los lugares (componentes del lugar) mediante figuras, nombres o símbolos (o una combinación de ellos). La técnica regularmente combina elementos de tipo cuantitativo y cualitativo para el análisis de la información obtenida. De lo primero sobresale el análisis estadístico descriptivo para identificar frecuencias de lugares, recurrencias, desviaciones, entre otros aspectos. De los segundos se destaca el análisis del discurso y la interpretación psicosocial de las imágenes descritas en el mapa.

La metodología aplicada regularmente implica tres momentos o fases:

1. Identificación del área de estudio (ciudad, barrio, sector, calle) y selección de los participantes.

2. Sistema de identificación. Consiste en aislar, describir e identificar las interacciones del sistema.

3. Recopilación de la información según el punto anterior. Análisis de la información utilizando los diversos instrumentos cuantitativos y cualitativos.

\section{C) Ventajas y desventajas}

Entre las ventajas se destaca el hecho de que es una técnica relativamente fácil de aplicar, exige pocos recursos y es económicamente viable en términos de tiempo de aplicación y costos. Sabiéndola aplicar, es una alternativa muy rica en información explícita e implícita y se puede utilizar con muy diversos propósitos para resolver problemas urbanos relacionados con la movilidad, el diseño de los espacios y hasta el análisis de riesgos.

La desventaja reside en que los sesgos en la información arrojada pueden ser muy grandes en dependencia de condiciones de tiempo, información y conocimiento disponible, experiencia de vida, e incluso por la ausencia o mala aplicación de estímulos para la participación de los habitantes de la ciudad.

Otra desventaja puede derivar de la falta de dominio de las técnicas y los métodos aplicables al análisis de la información.

\section{D) Ejemplos de aplicación}

La técnica de los mapas mentales, desde el trabajo pionero de Lynch (1960), se ha aplicado para analizar diversas representaciones del espacio urbano y de los elementos socioculturales que inciden en ellas, especialmente por medio de representaciones cartográficas de la ciudad en muy diversas investigaciones.

Por citar solo algunas:

García Ballesteros, Aurora y Joaquín Bosque Sendra (1989). El espacio subjetivo de la ciudad de Segovia. Madrid: UCM.

Estévez Adán, V. (1994). "Alcalá de Henares: Percepción de un espacio urbano según su población mayor”, Estudios Geográficos, t. LV, núm. 214, enero-marzo. 
Zárate Martín, A. y M. T. Rubio Benito (2002). Análisis de la ciudad. Espacio objetivo y espacio percibido. Proyecto multimedia de geografía humana. Madrid: UNED.

Figura 1

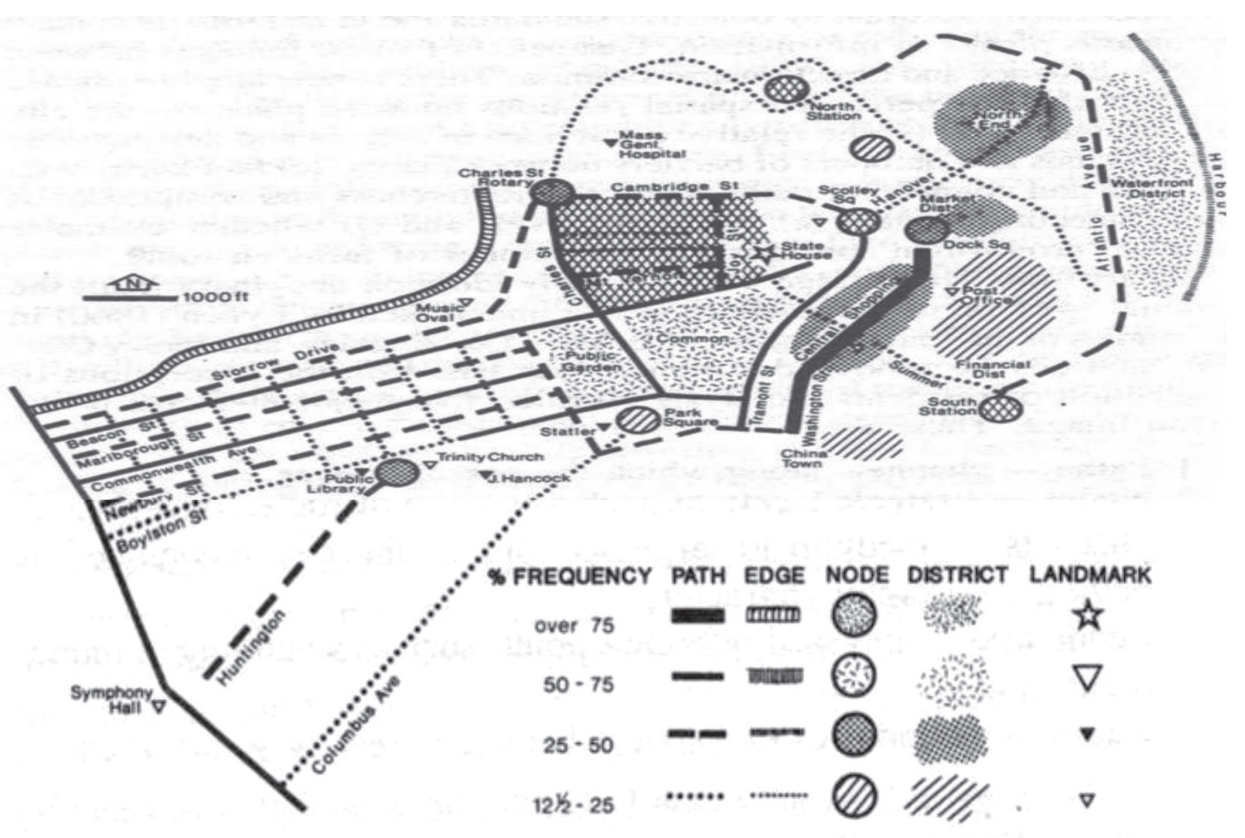

Source: Lynch 1960: 146

En México se han realizado diversas investigaciones sobre percepciones del espacio urbano en varias universidades del país, la UNAM, la UANL, UAM entre otras. En particular, una investigación sumamente interesante fue realizada por Martha de Alba, de la Universidad Autónoma Metropolitana Iztapalapa, y lleva por título Mapas mentales de la ciudad de México: Una aproximación psicosocial al estudio de las representaciones espaciales.

Las especificaciones técnicas de la investigación son las siguientes:

Objetivo del trabajo: Estudiar las representaciones cartográficas de la ciudad de México (DF) para conocer el contenido y la estructura de los mapas mentales de los residentes de la ciudad, así como los elementos culturales que los nutren; en particular, se pretende averiguar si el habitante de una urbe tan grande llega a formarse una representación del espacio urbano en su conjunto, si lo vive y lo concibe como un espacio continuo o fragmentado (p. 2). 


\section{Figura 2}

Imagen de la ciudad de Málaga, España

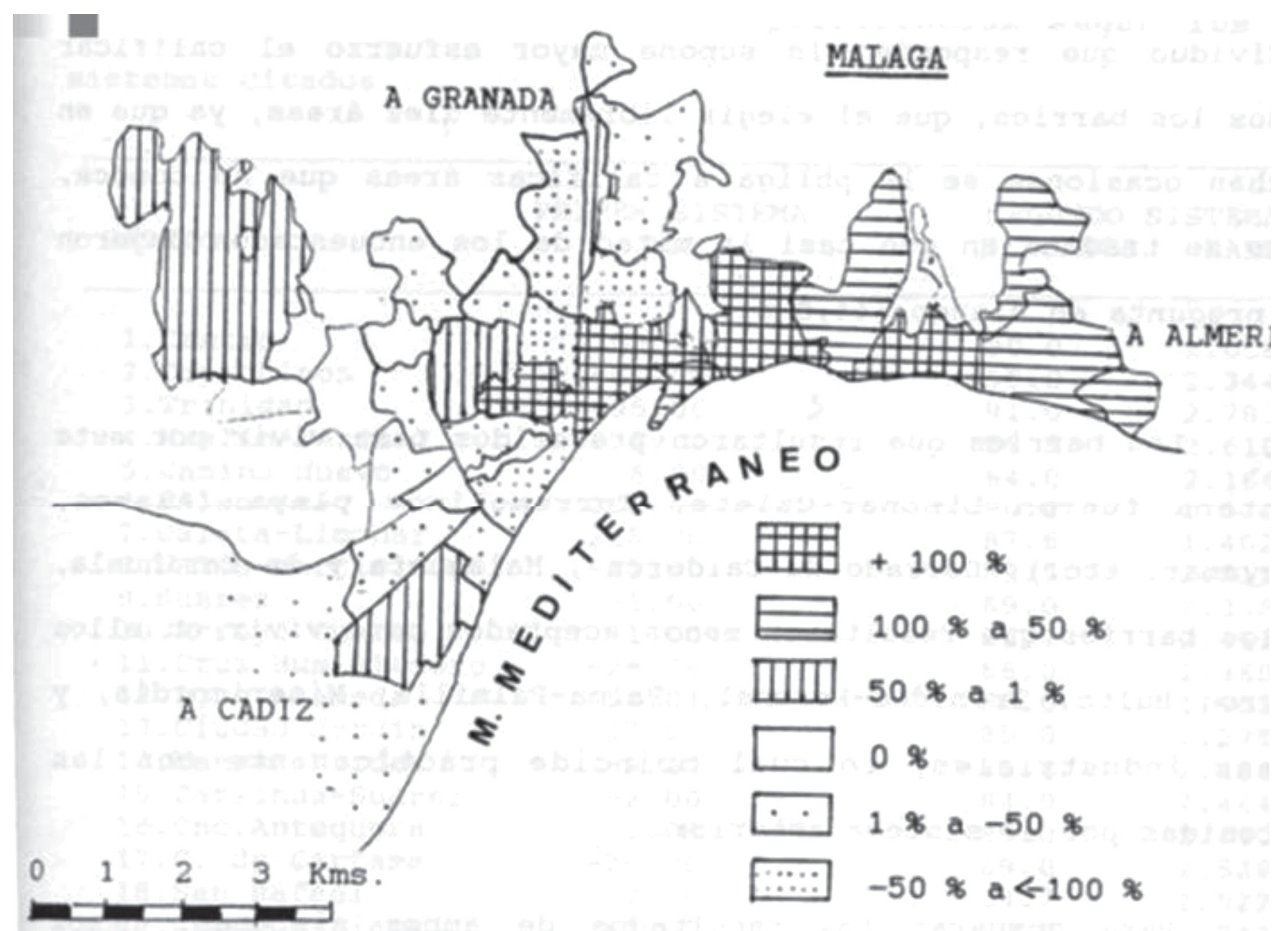

ESCALA

Fuente: "La geografía de la percepción: Análisis de las preferencias residenciales. Aplicación al municipio de Málaga” (1990), en las Actas del I Congreso de Jóvenes Historiadores y Geógrafos. Editorial de la Universidad Complutense de Madrid.

Metodología y universo de estudio: Se pidió a 60 entrevistados dibujar en una hoja blanca un mapa del espacio que se quería estudiar (en este caso la Ciudad de México). Participaron hombres y mujeres de entre 25 y 50 años, de nivel socioeconómico medio y con escolaridad mínima de bachillerato.

La actividad consistió en dibujar un mapa de la ciudad como ellos la imaginaban, señalando los lugares que iban dibujando. Una vez realizada la actividad de elaboración de mapas, con el propósito de apreciar la imagen colectiva de la ciudad se analizó el conjunto de mapas con un doble procedimiento: el análisis estadístico de las frecuencias de lugares dibujados (o denotados por su nombre), seguido de otro análisis mediante el programa del discurso conocido como ALCESTE (Análisis Lexical de Concurrencias en Enunciados Simples de un Texto). 
Resultados. No es el fin de este ensayo revisar de forma exhaustiva los resultados, baste con decir que se observan diferentes percepciones de los participantes, determinadas por las diferencias socioeconómicas, de edad, de escolaridad y por la experiencia y el conocimiento acumulado acerca de la ciudad. A pesar de ello, las percepciones colectivas de la ciudad quedan plasmadas en los mapas al considerar la recurrencia de los lugares citados por su valor simbólico, histórico y de identidad, como una muestra también de las diferencias socioculturales de la ciudad (p. 13). No fue casual que el bosque de Chapultepec, Xochimilco y el Centro Histórico fueran los lugares más referenciados en los mapas.

El estudio pone en evidencia las relaciones entre los procesos mentales de los participantes, los elementos físicos, la percepción de los habitantes y la connotación simbólica de los espacios. Deja constancia, de igual forma, de que el origen de las diferentes representaciones de la ciudad depende de las condiciones socioculturales de quienes la habitan, sin que ello impida la construcción colectiva de una imagen de la ciudad a partir de las representaciones recurrentes, como efectivamente ocurre con la ciudad de México en este estudio.

\section{Conclusiones}

No es tarea fácil atrapar o "aprehender" la complejidad, como apunta J. Flores (2013), mucho menos para un analfabeto en la materia, como lo es quien escribe estas líneas. Seguramente este artículo abonará a la confusión, y por qué no decirlo, la angustia que representa la falta de claridad en la exposición de temas tan "complejos", faltaba más, como el propio estudio de la complejidad.

Esta es la razón por la cual se inicia este ensayo tratando de delimitar lo que podría ubicarse en el entorno o marco de lo complejo, recurriendo a su antítesis, lo simple. Es así como el atrevimiento de quien esto escribe sintetizó la definición de la complejidad en ocho características, reconociendo que la primera de ellas me sirve como una especie de inmunidad intelectual, en el sentido en que E. Morín lo manifiesta con especial contundencia, "La complejidad es una palabra problema y no una palabra solución".

El estudio de los fenómenos que se muestran complejos, como el aquí abordado, constituye una oportunidad para reconocer que dichos fenómenos demandan la reflexión multidisciplinar y de saberes diversos. La necesaria convergencia de saberes no solo se refiere a los reconocidos por la epistemología positivista, sino también a aquellos sojuzgados y pretendidamente colonizados por dicha epistemología; de otra manera es difícil aproximarse al estudio de lo complejo.

Esta es la razón por la cual se propone en este ensayo el uso de un recurso extraordinario, e invaluablemente rico en información cualitativa, como es la literatura, en especial la narrativa, tal como se ilustra con la exposición de la obra de Í. Calvino Las ciudades invisibles, en cuyas interpretaciones, en principio fantásticas, se ven reflejadas muy diversas lecturas de lo que es la ciudad real o, mejor dicho, las muy diversas ciudades. 
En el mismo sentido, ilustrar el estudio de las representaciones sociales de la ciudad mediante el uso de un método, en apariencia simple pero complejo en su elaboración, los mapas mentales, se constituye en una alternativa para la investigación en ciencias sociales más allá de los límites que impone la ortodoxia epistemológica, de la cual, sin duda, se nutren la racionalidad instrumental y el individualismo metodológico tan característicos de la investigación en economía convencional.

Reconociendo la osadía que implica incursionar en el estudio de la complejidad, no me queda más que reiterar que este ensayo no pretende ser algo más que una invitación para romper con las ataduras que trata de imponer el mal llamado "pensamiento único" en economía y ciencias sociales.

\section{Referencias}

Amendola, Giandomenico (2000). La ciudad postmoderna. Magia y miedo de la metrópoli contemporánea. España: Celeste Ediciones.

Cabrera, D. H. (s/f). Imaginario social, comunicación e identidad colectiva. Disponible en: www.portalcomunicacion.com/dialeg/paper/pdf/143-cabrera.pdf.

Calvino, Í. (1999). Las ciudades invisibles. Colección las 100 joyas del Milenio. México DF: Grupo Editorial Milenio.

Caravaca Morera, J. A (2017). "Las representaciones sociales y la cognición social: contribuciones para la investigación en enfermería y salud". Texto Contexto, 26(4). Disponible en: http://dx.doi.org/10-1590/0104-07072017001500017.

Castoriadis, C. (1975). La institución imaginaria de la sociedad. Buenos Aires: Tusquets.

Cocho, G. y G. Martínez Mekler (s/f). Complejidad en comportamientos universales en las artes y las ciencias.

Díaz, J. L. (s/f). El cerebro, enjambre de la conciencia.

Flores Valdés J. y G. Martínez Mekler (2013) (Comp.). Encuentros con la complejidad. México, DF: Siglo XXI-UNAM.

Flores, J. (s/f). Para atrapar la complejidad.

García Canclini, N. (1999). La globalización imaginada. Buenos Aires: Paidós.

Hovellebecq, M. (2011). El mapa y el territorio. Barcelona: Anagrama.

Jodelet, D. (1986). "La representación social: fenómenos, concepto y teoría". En S. Moscovici (Comp.), Psicología social. Barcelona: Paidós.

Jonhston, R. J. (1971). "Mental Maps of the City: Suburban Preference Patterns". Rev. Environment and Planning, vol. 3, pp. 63-72.

Lefebvre, H. (1969). El derecho a la ciudad. Barcelona: Península.

Lizcano, E. (2003). "Imaginario colectivo y análisis metafórico". Conferencia inaugural del Primer Congreso Internacional de Estudios sobre Imaginario y Horizontes Culturales, celebrado en la Universidad Autónoma del Estado de Morelos, Cuernavaca, México, del 6 al 9 de mayo de 2003. 
Lynch, Kevin (2008[1960]). La imagen de la ciudad. Barcelona: Gustavo Gili. [De la versión en inglés The Image of the City. The Massachusetts Institute of Technology Press].

Martínez Mekler, G. (2000). "Una aproximación a los sistemas complejos”. Ciencias Revista de Difusión. Facultad de Ciencias. México, DF: UNAM.

Morales, O. G. (2014). Representaciones de alteridades "negras", africanas y afrodescendientes, en la sociedad nacional en Argentina, primera década del siglo XXI. Tesis de doctorado. Universidad Nacional de la Plata. Disponible en: http://sedici.unlp. edu.ar/handle/10915/39073.

Moscovici, S. (1961). El psicoanálisis, su imagen y su público. Buenos Aires: Huemal.

Nudler, O. (2009). Espacios controversiales. Hacia un modelo de cambio filosófico y científico Buenos Aires: Miño y Dávila.

Park, R. E. (1999). La ciudad y otros ensayos de ecología urbana. Barcelona: Ediciones del Serbal.

Reza, G. A. de la (2010). Sistemas complejos. Perspectivas de una teoría general. México, DF: Antropos, Universidad Autónoma Metropolitana, Azcapotzalco.

Safa, P. (2000). "El estudio de las identidades vecinales: una propuesta metodológica". Rev. Universidad de Guadalajara, núm. 18. Guadalajara, Jalisco, México.

Simmel, G. (1986). El individuo y la libertad. Ensayos de crítica de la cultura. Barcelona: Península.

Soto González, M. (1999). Edgar Morin. Complejidad y sujeto humano. Tesis doctoral. Universidad de Valladolid, Departamento de Filosofía, Lógica y Filosofía de la Ciencia y Teoría de la Educación. Disponible en: http://www.cervantesvirtual.com/ descargaPdf/edgar-morin-complejidad-y-sujeto-humano.

Stephens Ch. R. (s/f). Lo que no es la complejidad. 